\title{
Association between family history, early growth and the risk of beta cell autoimmunity in children at risk for type 1 diabetes
}

\author{
Danièle Pacaud $^{1}$ (D) - Anita M. Nucci ${ }^{2}$ (D) $\cdot$ David Cuthbertson $^{3}$ (D) Dorothy J. Becker $^{4}$ (D) Suvi M. Virtanen ${ }^{5,6,7,8}$ (D) \\ Johnny Ludvigsson ${ }^{9}$ (D) . Jorma llonen ${ }^{10,11}$ (D) $\cdot$ Mikael Knip ${ }^{12,13}$ (D) $\cdot$ the TRIGR investigators
}

Received: 24 April 2020 / Accepted: 7 August 2020 / Published online: 7 October 2020

(C) The Author(s) 2020

\begin{abstract}
Aims/hypothesis The aim of this work was to examine the relationship between family history of type 1 diabetes, birthweight, growth during the first 2 years and development of multiple beta cell autoantibodies in children with a first-degree relative with type 1 diabetes and HLA-conferred disease susceptibility.

Methods In a secondary analysis of the Trial to Reduce IDDM in the Genetically at Risk (TRIGR), clinical characteristics and development of beta cell autoantibodies were compared in relation to family history of type 1 diabetes (mother vs father vs sibling) in 2074 children from families with a single affected family member.

Results Multiple autoantibodies ( $\geq 2$ of 5 measured) developed in 277 (13\%) children: 107 (10\%), 114 (16\%) and 56 (18\%) born with a mother, father or sibling with type 1 diabetes, respectively $(p<0.001)$. The HR for time to multiple autoimmunity was 0.54 $(95 \%$ CI $0.39,0.75)$ in offspring of affected mothers $(n=107 / 1046, p<0.001)$ and $0.81(95 \%$ CI $0.59,1.11)(n=114 / 722, p=0.19)$ in offspring of affected fathers, compared with participants with a sibling with type 1 diabetes (comparator group $n=56 / 306$ ). The time to the first autoantibody present (to insulin, GAD, tyrosine phosphatase-related insulinoma-associated 2 molecules, islet cell or zinc transporter 8 ) was similar in the three groups. Height velocity ( $z$ score/year) in the first 24 months was independently associated with developing multiple antibodies in the total cohort (HR 1.31 [95\% CI 1.01, 1.70], $p=0.04$ ). A higher birthweight in children born to an affected mother vs affected father or an affected sibling was not related to the risk of multiple autoimmunity.

Conclusions/interpretation The risk of developing multiple autoantibodies was lower in children with maternal type 1 diabetes. For the whole group, this risk of developing multiple autoantibodies was independent of birthweight but was greater in those with increased height velocity during the first 2 years of life. However, the risk associated with paternal type 1 diabetes was not linked to differences in birthweight or early growth.

Trial registration ClinicalTrials.gov NCT00179777
\end{abstract}

Keywords Beta cell autoimmunity $\cdot$ Birthweight $\cdot$ Familial risk $\cdot$ Father $\cdot$ Genetic risk $\cdot$ Linear growth $\cdot$ Mother $\cdot$ Proband $\cdot$ Type 1 diabetes

The TRIGR investigators are listed in the electronic supplementary material (ESM).

Electronic supplementary material The online version of this article (https://doi.org/10.1007/s00125-020-05287-1) contains peer-reviewed but unedited supplementary material, which is available to authorised users.

Danièle Pacaud

daniele.pacaud@ahs.ca

Mikael Knip

mikael.knip@helsinki.fi

Extended author information available on the last page of the article

\section{Abbreviations}

GADA GAD autoantibodies

GLM General linear regression model

IAA Insulin autoantibodies

IA-2A Tyrosine phosphatase-related insulinoma-associated 2 molecule autoantibodies

ICA Islet cell autoantibodies

TEDDY The Environmental Determinants of Diabetes in the Young

TRIGR Trial to Reduce IDDM in the Genetically at Risk

ZnT8A Zinc transporter 8 autoantibodies 


\section{Research in context}

What is already known about this subject?

- The risk of type 1 diabetes in the offspring is increased in paternal compared with maternal diabetes

- Increased birthweight and rapid linear growth during infancy are reported to be related to development of type 1 diabetes

What is the key question?

- In children with a positive family history, is the difference in risk of developing type 1 diabetes-associated autoantibodies between children with a father, mother or sibling with type 1 diabetes related to birthweight and postnatal growth during the first 2 years?

What are the new findings?

- In this large group of children with familial risk, we observed that differing risk of developing autoantibodies based on family history was not linked to birthweight or growth during the first 2 years

How might this impact on clinical practice in the foreseeable future?

- A better understanding of the pathogenesis of diabetes-related autoantibodies may help to provide individualised risk stratification and eventually tailor preventive measures for children at risk of type 1 diabetes

\section{Introduction}

Type 1 diabetes is one of the most prevalent chronic diseases in childhood. Its incidence is rapidly increasing worldwide [1-5]. Type 1 diabetes is caused by a progressive but heterogeneous autoimmune destruction of the beta cells resulting in lack of insulin. Both genetic and environmental factors contribute to this process, although its pathophysiology is poorly understood [6]. Historically, the risk of diabetes in the offspring of fathers with type 1 diabetes has been reported as being higher than that in the offspring of mothers with type 1 diabetes or in those with siblings who have type 1 diabetes [7]. Many researchers have examined the link between birthweight and risk of either type 1 diabetes or beta cell autoimmunity with variable results. This may reflect the heterogeneity of the sample size, study population and methodology used. However, a large meta-analysis of 29 studies predominantly from European centres, found that the risk of type 1 diabetes was significantly higher in children with greater birthweights [8]. Larger studies, looking specifically at individuals genetically at risk for type 1 diabetes, observed that greater birthweight is related to development of type 1 diabetes $[9,10]$ or beta cell autoimmunity $[10]$ and that this pattern is similar across different geographical regions. Furthermore, some studies that were able to prospectively observe children with genetic risk for type 1 diabetes have identified rapid linear growth during infancy as a risk factor for the development of beta cell autoimmunity and type 1 diabetes [11-14].

Although The Environmental Determinants of Diabetes in the Young (TEDDY) study group recently reported that family history is not a significant risk factor for the progression to diabetes in individuals who develop multiple autoantibodies
[15], their previous reports focusing on growth and risk beta cell autoimmunity, did not account for first-degree relative status in their analysis [16]. However, a recent analysis examining the effect of interactions between genetic and environmental factors on the development of islet cell autoimmunity and diabetes indicated that family history (father or sibling vs no family history) significantly influenced both, while weight at 12 months only affected development of autoimmunity but not type 1 diabetes [17]. To our knowledge, none of the studies looking at birthweight, infant growth, HLA typing and risk of beta cell autoimmunity evaluated possible differences in risk linked to birthweight, infant growth and autoimmunity associated with the proband (father, mother or sibling). In fact, many of these studies excluded maternal diabetes from the analysis in order to avoid the confounding factor associated with maternal metabolic control and birthweight. In this study we examined the relationship between the family history of type 1 diabetes (mother vs father vs sibling), birthweight and initial 2 year growth and autoimmunity in a cohort of 2074 participants with one first-degree relative with type 1 diabetes in the Trial to Reduce IDDM in the Genetically at Risk (TRIGR) study (ClinicalTrials.gov registration no. NCT00179777).

\section{Methods}

\section{Study design and participants}

The TRIGR study was an international double-blinded clinical trial designed to determine whether weaning to a hydrolysed 
infant formula vs a conventional cow's milk-based formula reduces the cumulative incidence of diabetes-associated autoantibodies and the incidence of diabetes in young children with a first-degree relative with type 1 diabetes and increased HLA-conferred genetic risk. The trial was approved in all participating TRIGR centres by the Ethics Institutional Review Boards or Committees of Human Experimentation and informed consent was obtained for each participating child. A full description of the TRIGR study design has been published previously [18]. Children at increased risk for developing type 1 diabetes were recruited for the trial before or at birth between May 2002 and January $2007(n=2159)$ and were monitored until February 2017, when all children were at least 10 years of age, for the frequency of beta cell autoantibodies and/or the development of type 1 diabetes. For the purpose of this secondary analysis, we excluded 73 children with more than one first-degree family member with type 1 diabetes: ten with mother and sibling; 33 with father and sibling; 29 with both mother and father; and one with a mother, father and sibling with type 1 diabetes. Another four were excluded because of missing family relationship status and eight were excluded due to missing antibody data.

Data collection Only data collected during the conduct of the original TRIGR study was used for this secondary analysis. Demographic characteristics, including sex, race, ethnicity and data on type 1 diabetes in first-degree relatives, were obtained at the time of enrolment or birth. HLA genotyping to determine eligibility was performed [19]. Anthropometric indices were obtained at birth and at regular intervals (values at 3, 6, 9, 12, 18 and 24 months of age are included in this analysis). Weight velocity (kg/year) and length/height velocity (cm/year), as well as corresponding $z$ score values calculated from 2000 CDC growth charts [20], were recorded for the first 2 years of life. BMI $\left(\mathrm{kg} / \mathrm{m}^{2}\right)$ was calculated at 2 years of age. The definition of exclusive breastfeeding was intake of only breast milk (banked or mother's own), supplementary vitamins or minerals or water. The presence of beta cell autoantibodies was determined in a central laboratory (Scientific Laboratory, Children's Hospital, University of Helsinki, Helsinki, Finland) at birth and at 3, 6, 9, 12, 18 and 24 months of age and annually thereafter. Islet cell autoantibodies (ICA) were measured using indirect immunofluorescence with a cutoff of 2.5 JDRF units [19] and GAD autoantibodies (GADA), tyrosine phosphatase-related insulinoma-associated 2 molecule autoantibodies (IA-2A), insulin autoantibodies (IAA) and zinc transporter 8 autoantibodies (ZnT8A) were analysed with specific radiobinding assays with cut-off limits of 5.36 RU, 0.77 RU, 2.80 RU and 0.61 RU, respectively [21]. Development of an autoantibody was defined as the first occurrence of a result above the specified cut-off limits. The disease-specific sensitivity and specificity for each were previously reported [19, 22]. Maternal antibodies that were placentally transferred, as verified by their decreasing levels and disappearance from the child's circulation by age 18 months, were not included in the statistical analysis.

\section{Statistical analysis}

We compared demographic, clinical and anthropometric characteristics by family history of type 1 diabetes (mother vs father vs sibling) univariately using the $\chi^{2}$ test for categorical variables and ANOVA for continuous variables. Cox proportional hazard univariate model was used to analyse the relationship between family history of type 1 diabetes (mother vs father vs sibling) and time to multiple autoimmunity (defined as two or more autoantibodies), positivity for GADA, IAA, IA-2A, ICA and ZnT8A and development of clinical type 1 diabetes. Multivariate Cox proportional hazard regression models were created to predict time to antibodies (multiple autoantibodies, GADA, IAA, IA-2A, ICA or ZnT8A) and the development of clinical type 1 diabetes in each family history group (mother vs father vs sibling). The following variables thought to be clinically important were selected a priori: country of birth, sex, HLA risk group, mode of delivery, birth length, birthweight, treatment group, height velocity in the first 24 months of life (cm/year), weight velocity in first 24 months of life ( $z$ score), BMI $z$ score at 24 months of life, infant diet in the first days of life, and length of exclusive breastfeeding. These were then included in a stepwise model when found to be statistically significant. Using the same covariates listed above, general linear regression models (GLMs) were used to examine the difference between the three groups of origin of the proband in linear growth both in $\mathrm{cm} /$ year and $z$ score/year. All HRs are presented with the sibling group as the reference group. A $p$ value of $<0.05$ was considered statistically significant for the comparisons between the three groups. All participants with missing data specified as part of a particular analysis were omitted from that analysis. No imputation of data was performed. All analyses were conducted using SAS version 9.4, USA. All results presented in the Results section are new and were not part of previous TRIGR reports.

\section{Results}

Beta cell autoimmunity and relationship to family history of type 1 diabetes The clinical characteristics of the 2074 children with a single first-degree relative with type 1 diabetes are presented in Table 1. During the study period, positive seroconversion occurred in $933(45 \%)$ children, which included 346 (17\%) with GADA, 319 (15\%) with IAA, 196 (9\%) with IA-2A, $724(35 \%)$ with ICA and 171 (8\%) with ZnT8A. Multiple autoantibodies (two or more of GADA, IA-2A, IAA, ZnT8A and ICA) developed in 277 (13\%) children: 
$107(10 \%), 114(16 \%)$ and $56(18 \%)$ of children born with a mother, father or sibling with type 1 diabetes, respectively. Time to the development of multiple autoantibodies in the child differed significantly between those with an affected mother, father or sibling (time to $10 \%$ cumulative incidence $[95 \% \mathrm{CI}]$ : mothers 9.3 years $[7.7,11.3]$; fathers 6.3 years $[5.2$, $7.6]$; sibling 5.0 years $[3.9,6.5])\left(\operatorname{logrank} \chi^{2} 16.2, p<0.001\right)$ (Fig. 1a). Pairwise comparisons between the three groups indicated that while there was not a significant difference in the progression to multiple autoantibodies between those with an affected father and sibling (logrank $\chi^{2} 1.7, p=0.189$ ), there was a significant difference between those with an affected mother and, respectively, those with an affected father (logrank $\chi^{2} 8.7, p=0.003$ ) and those with an affected sibling (logrank $\left.\chi^{2} 14.0, p<0.001\right)$.

First positive beta cell autoantibody and relationship of family member affected by type 1 diabetes In children developing multiple antibodies, the first was GADA in $52(19 \%)$, IAA in $48(18 \%)$, ICA in 46 (17\%), IA-2A in three (1\%) and ZnT8A in three $(1 \%)$. Multiple antibodies occurred in the first positive sample in 119 (44\%). Among the 119 children who developed multiple antibodies in the first sample, $59 \%$ tested positive for GADA, 52\% for IAA, $62 \%$ for ICA, $27 \%$ for IA-2A and $21 \%$ for $\mathrm{ZnT} 8 \mathrm{~A}$, respectively. There was no difference in which antibody appeared first between the three groups $(p=0.39)$. Times to each antibody as the first antibody present were similar in all three groups (logrank $\chi^{2} 0.5-5.5$; all $p>0.05$ ) (Fig. 1b-f).

\section{Association between birth and growth variables and affected} family member relationship Despite early differences in mode of delivery, gestational age, birthweight, birth length and duration of exclusive breastfeeding (see Table 1), univariate analysis demonstrated at age 24 months only mild non-significant differences in BMI $z$ score (ESM Fig. 1, $p=0.054$ ), height velocity (ESM Fig. 2a, $p=0.053$ ) between the three groups. However, there was a significant difference between the three groups in height velocity $z$ score (ESM Fig. 2b, $p=0.041$ ), explained by a wider variation in growth seen in children born to a father with diabetes. GLMs predicting height velocity either as cm/year (Model 1, ESM Table 1) or $z$ score per year (Model 2, ESM Table 1) until age 24 months by relative with type 1 diabetes resulted in significant differences: height velocity in $\mathrm{cm} /$ year $R^{2}$ was 0.246 with $F(13,1754)=44.0$; $p<0.001$, while for height velocity in $z$ score/year $R^{2}$ was 0.399 with $F(13,1754)=89.5 ; p<0.001$. Variables found to contribute to each model were similar between the two GLM models (birth length, birthweight, mother with type 1 diabetes, region of origin, duration of exclusive breast feeding, being fed only infant formula in the first week of life vs other types of first diet, birth order and gestational age) (ESM Table 1). In both models, the relative with type 1 diabetes showed a statistically significant but minor association: infants of an affected mother explained $<1 \%$ of the variation in growth. Re-enforcing this point, removing family history as a variable in these models produced remarkably similar results, with $R^{2}$ values of 0.237 and 0.391 for the models for height velocity in $\mathrm{cm} /$ year and $z$ score/year, respectively).

Association between the development of multiple autoantibodies, family member with type 1 diabetes and first 2 years of physical growth When Cox proportional hazard stepwise multivariate modelling for time to multiple autoimmunity with an a priori inclusion of family member with type 1 diabetes was used, being born to a mother with type 1 diabetes remained the lowest risk of developing multiple antibodies (HR 0.70 [95\% CI 0.49, 1.01], $p=0.055$ ) compared with an affected father or sibling (Table 2). The other significant variables retained in this model were HLA risk 1 (HR 1.74 [95\% CI 1.33, 2.28], $p<0.001$ ), birth length (HR 1.09 [95\% CI $1.03,1.16], p=0.004)$ and height velocity $z$ scores in the first 24 months (HR 1.31 [95\% CI 1.01, 1.70], $p=0.041$ ).

Association between risk of type 1 diabetes and relationship of affected family member The time to the development of type 1 diabetes was compared between the three groups of family member with type 1 diabetes. Univariate analyses indicated that there was an overall difference between the groups (time to $5 \%$ cumulative incidence $[95 \% \mathrm{CI}]$ : mothers 10.0 years [7.7, 12.9]; fathers 6.9 years [5.4, 8.9]; sibling 4.3 years $[3.1,5.9])\left(\operatorname{logrank} \chi^{2} 17.2, p<0.001\right)(\mathrm{ESM}$ Fig. 3). When Cox proportional hazard stepwise multivariate modelling for time to development of type 1 diabetes with an a priori inclusion of family member with type 1 diabetes was used, those with a mother, compared with an affected father or sibling, with type 1 diabetes remained at lowest risk of developing type 1 diabetes (HR 0.58 [95\% CI $0.36,0.93], p=0.02)$. The other significant variables retained in this model were HLA risk 1 (HR 2.21 [95\% CI $1.54,3.16)], p<0.001)$ and height velocity $z$ score in the first 24 months (HR 1.41 [95\% CI 1.10, 1.82], $p$ $<0.01$ ) (Table 3).

\section{Discussion}

In this large international cohort of children genetically at risk for type 1 diabetes followed for more than 8 years, we confirmed prior data showing that the risk of developing multiple autoantibodies was lower when children were born to mothers with type 1 diabetes compared with those having an affected father [23] or an affected sibling [24]. We were able to search for potential differences in demographics or patterns of autoimmunity that could help explain this 
Table 1 Demographic, clinical and anthropometric characteristics by family history of type 1 diabetes (mother vs father vs sibling)

\begin{tabular}{|c|c|c|c|c|}
\hline Variable & $\begin{array}{l}\text { Mother } \\
(n=1046)\end{array}$ & $\begin{array}{l}\text { Father } \\
(n=722)\end{array}$ & $\begin{array}{l}\text { Sibling } \\
(n=306)\end{array}$ & $\begin{array}{l}p \\
\text { value }\end{array}$ \\
\hline Male sex, $n(\%)$ & $547(52)$ & $391(54)$ & $156(51)$ & NS \\
\hline Birth order ${ }^{a}$ & $1(1)$ & $1(1)$ & $2(1)$ & $<0.001$ \\
\hline Birthweight, kg & $3.65 \pm 0.60$ & $3.56 \pm 0.49$ & $3.57 \pm 0.49$ & 0.004 \\
\hline Birth length, $\mathrm{cm}$ & $50.9 \pm 3.0$ & $51.0 \pm 2.4$ & $51.6 \pm 2.7$ & $<0.001$ \\
\hline Weight at 24 months, $\mathrm{cm}$ & $12.8 \pm 1.6$ & $12.9 \pm 1.4$ & $12.9 \pm 1.7$ & 0.627 \\
\hline Weight at 24 months, $z$ score & $0.2 \pm 1.1$ & $0.3 \pm 1.0$ & $0.2 \pm 1.2$ & 0.346 \\
\hline BMI at 24 months, $z$ score & $0.08 \pm 1.15$ & $-0.03 \pm 1.04$ & $-0.15 \pm 1.17$ & 0.054 \\
\hline Height velocity at 24 months, $\mathrm{cm} /$ year & $18.3 \pm 2.0$ & $18.5 \pm 1.9$ & $18.2 \pm 1.8$ & 0.053 \\
\hline Height velocity at 24 months, $z$ score & $-0.04 \pm 0.66$ & $0.00 \pm 0.61$ & $-0.011 \pm 0.56$ & 0.041 \\
\hline${ }^{\mathrm{b}}$ HLA risk type, $n(\%)$ & & & & 0.017 \\
\hline HLA risk 1 & $229(22)$ & $171(24)$ & $95(31)$ & \\
\hline HLA risk 2 & $456(44)$ & $336(47)$ & $117(38)$ & \\
\hline HLA risk 3 & $350(34)$ & $210(29)$ & $92(30)$ & \\
\hline HLA risk 4 & $11(1)$ & $5(1)$ & $2(1)$ & \\
\hline $\begin{array}{l}\text { Length of exclusive breastfeeding, } \\
\text { months }\end{array}$ & $0.7 \pm 1.6$ & $1.8 \pm 2.2$ & $2.3 \pm 2.3$ & $<0.001$ \\
\hline Caesarean section, $n(\%)$ & $642(61.4)$ & $183(25.3)$ & $74(24.2)$ & $<0.001$ \\
\hline Gestational age, weeks & $37.8 \pm 1.3$ & $39.8 \pm 1.4$ & $39.4 \pm 1.4$ & $<0.001$ \\
\hline \multicolumn{5}{|l|}{ Autoantibodies, $n(\%)$} \\
\hline Multiple & $107(10)$ & $114(16)$ & $56(18)$ & $<0.001$ \\
\hline IAA & $139(13)$ & $110(15)$ & $70(23)$ & $<0.001$ \\
\hline GADA & $154(15)$ & $126(18)$ & $66(22)$ & 0.015 \\
\hline IA-2A & $72(7)$ & $85(12)$ & $39(13)$ & $<0.001$ \\
\hline ZnT8A & $65(6)$ & $75(10)$ & $31(10)$ & 0.003 \\
\hline ICA & $337(32)$ & $270(37)$ & $117(38)$ & 0.034 \\
\hline Type 1 diabetes, $n(\%)$ & $59(6)$ & $60(8)$ & $38(12)$ & $<0.001$ \\
\hline Follow-up time, years & $9.1 \pm 4.3$ & $9.4 \pm 4.0$ & $8.7 \pm 4.3$ & 0.032 \\
\hline
\end{tabular}

Values are expressed as mean $\pm \mathrm{SD}$ or $n(\%)$

${ }^{\text {a }}$ Values are expressed as median (IQR)

${ }^{\mathrm{b}}$ HLA risk 1, HLA-DQB $* 02 / D Q B 1 * 03: 02$; HLA risk 2, $H L A-D Q B 1 * 03: 02 / x$ (x not $D Q B 1 * 02, D Q B 1 * 03: 01$ or $D Q B 1 * 06: 02$ ); HLA risk 3, HLA-DQA1*05-DQB1*02/y (y not $D Q A 1 * 02: 01-D Q B 1 * 02, D Q B 1 * 03: 01$, $D Q B 1 * 06: 02$ or $D Q B 1 * 06: 03$ ); HLA risk 4, HLA-DQA1*03-DQB1*02/y (y not $D Q A 1 * 02: 01-D Q B 1 * 02$, $D Q B 1 * 03: 01, D Q B 1 * 06: 02$ or $D Q B 1 * 06: 03)$ difference. We observed no difference in the type of autoantibody appearing first between the three groups.

Despite differences in birth demographics among these three groups of relatives, this differential risk was not related to birthweight or linear growth (height and BMI) in the first 24 months of life. In single correlations, the children born to a mother with type 1 diabetes did have a higher birthweight and lower birth length. Based on the prediction growth models, the children with higher birthweight and lower birth length grew faster in the first 24 months. Maternal diabetes had a negative but minimal impact on growth during the first 24 months. Hence, based on the growth models, we would have expected these children to have increased risk of developing multiple autoantibodies. Yet, the Cox proportional hazard stepwise multivariate modelling for time to multiple autoimmunity showed that although those with increased birth length and growth velocity developed multiple autoantibodies sooner, children born to a mother with type 1 diabetes were less likely to develop multiple autoantibodies. Because of the differing results of the single correlations and the two predictive models, we conclude that the risk related to family history was not explained by differences in birthweight or growth during the first 2 years between the groups.

Three studies that specifically evaluated differences in number and type of measured autoantibodies in offspring of mothers compared with fathers with type 1 diabetes also reported that children with a father with type 1 diabetes were more likely to develop multiple autoantibodies than those with a mother with diabetes $[23,25,26]$. In contrast to our TRIGR study, these three studies did not account for HLA type risk 
a

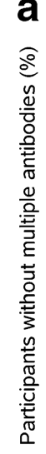

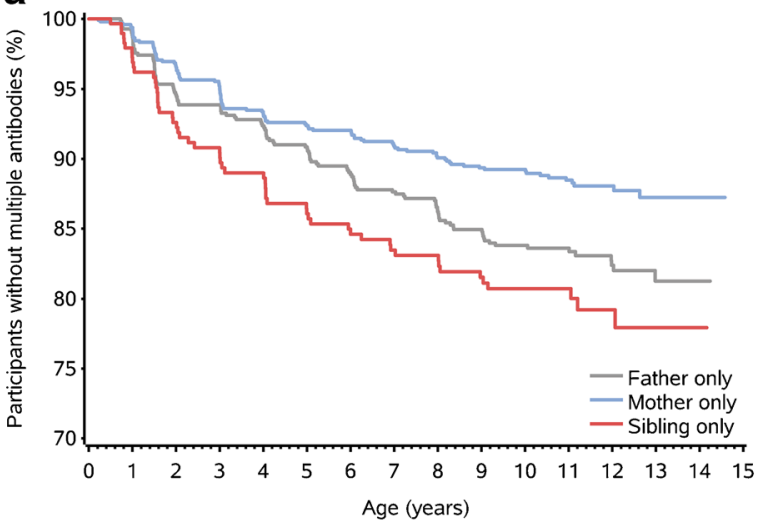

Number at risk:

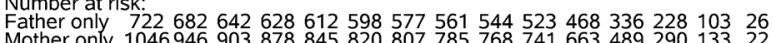

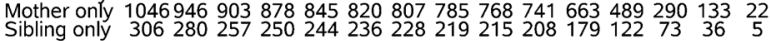

\section{C}

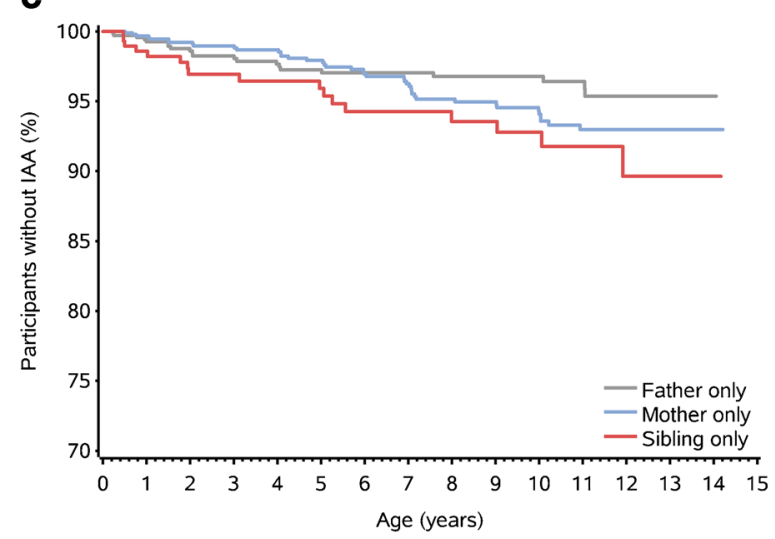

Number at risk:

Father only $722643571528485456427392364344294207134 \quad 49 \quad 9$

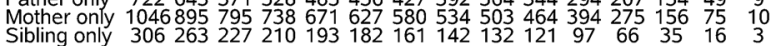

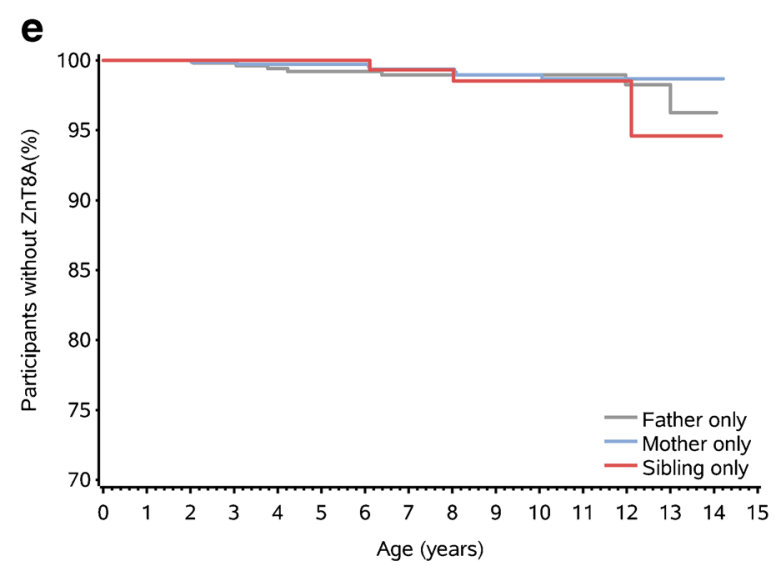

Number at risk

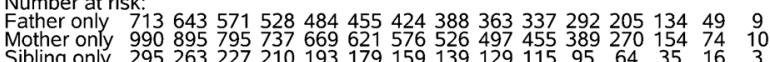

Fig. 1 Time to presence of autoantibodies by family history (mother vs father vs sibling): multiple autoantibodies defined as two or more antibodies (logrank $\left.\chi^{2} 16.2, p<0.001\right)(\mathbf{a})$; GADA as first antibody (logrank $\left.\chi^{2} 4.8, p=0.090\right)(\mathbf{b})$; IAA as first antibody (logrank $\left.\chi^{2} 5.5, p=0.064\right)$ b

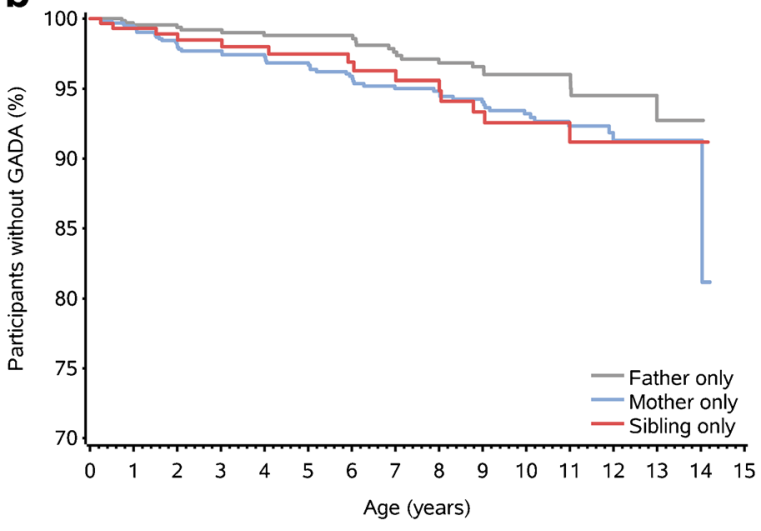

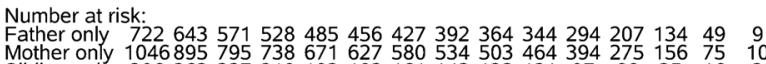

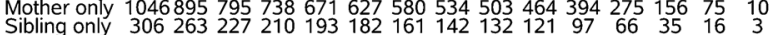

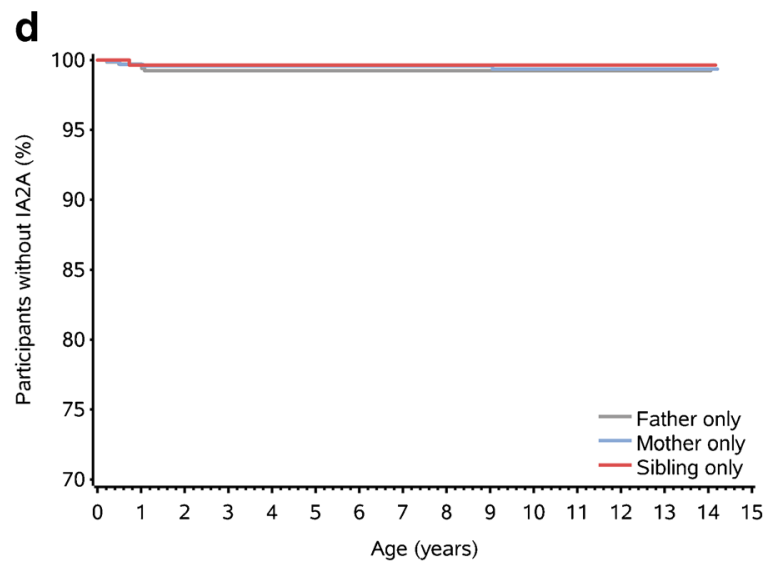

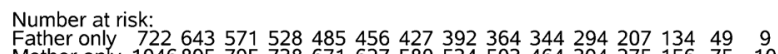

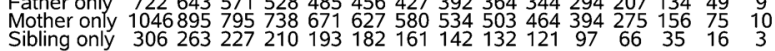

\section{f}

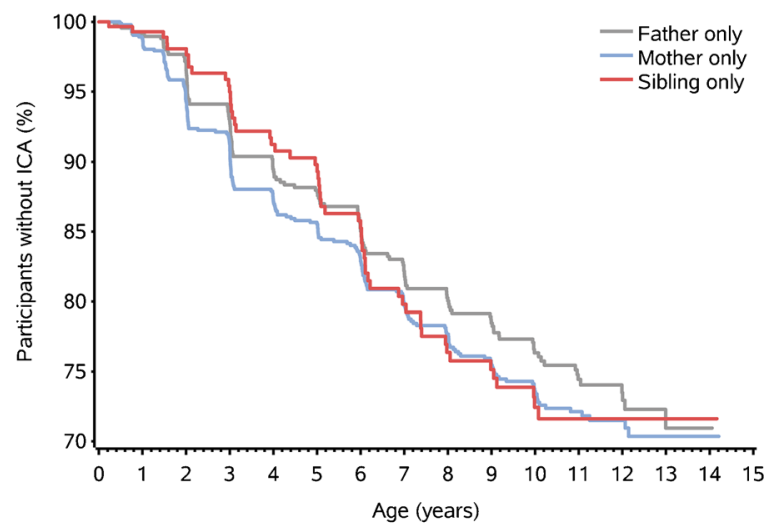

Number at risk:

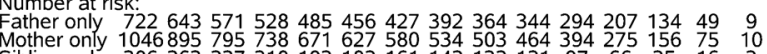
Sibling only $306263227210193182161142 \quad 132 \quad 121 \quad 97 \quad 66 \quad 35 \quad 16 \quad 3$

(c); IA-2A as first antibody (logrank $\chi^{2} 0.55, p=0.761$ ) (d); ZnT8A as first antibody (logrank $\left.\chi^{2} 0.6, p=0.73\right)(\mathbf{e})$; and ICA as first antibody $\left(\log a n k \chi^{2} 1.2, p=0.55\right)(\mathbf{f})$ 
Table 2 HR (95\% CI) from univariate and multivariate Cox time to development of multiple autoantibodies model

\begin{tabular}{|c|c|c|c|c|}
\hline \multirow[t]{2}{*}{ Variable } & \multicolumn{2}{|l|}{ Univariate models } & \multicolumn{2}{|l|}{ Multivariate model } \\
\hline & $\mathrm{HR}(95 \% \mathrm{CI})$ & $\begin{array}{l}p \\
\text { value }\end{array}$ & $\mathrm{HR}(95 \% \mathrm{CI})$ & $\begin{array}{l}p \\
\text { value }\end{array}$ \\
\hline Family history from mother & $0.54(0.39,0.75)$ & $<0.001$ & $0.70(0.49,1.01)$ & 0.055 \\
\hline Family history from father & $0.81(0.59,1.11)$ & 0.191 & $0.93(0.65,1.33)$ & 0.682 \\
\hline HLA risk $1^{\mathrm{a}}$ & $1.92(1.50,2.45)$ & $<0.001$ & $1.74(1.33,2.28)$ & $<0.001$ \\
\hline Birth length & $1.06(1.01,1.11)$ & 0.012 & $1.09(1.03,1.16)$ & 0.004 \\
\hline Height velocity ( $z$ score) & $1.03(0.84,1.27)$ & 0.758 & $1.31(1.01,1.70)$ & 0.041 \\
\hline Weight velocity ( $z$ score) & $1.02(0.84,1.23)$ & 0.880 & & \\
\hline Male sex & $1.27(1.00,1.61)$ & 0.054 & & \\
\hline Birthweight & $1.08(0.87,1.33)$ & 0.494 & & \\
\hline Gestational age, weeks & $1.05(0.98,1.13)$ & 0.168 & & \\
\hline \multicolumn{5}{|l|}{ Region of origin } \\
\hline Northern Europe & $1.11(0.85,1.45)$ & 0.449 & & \\
\hline Central Europe & $1.10(0.76,1.58)$ & 0.614 & & \\
\hline Vaginal delivery & $1.24(0.97,1.58)$ & 0.084 & & \\
\hline $\begin{array}{l}\text { Treatment group assignment casein hydrosylate } \\
\text { formula }\end{array}$ & $1.13(0.89,1.43)$ & 0.314 & & \\
\hline Exclusive breastfeeding duration & $1.05(1.00,1.11)$ & 0.071 & & \\
\hline Birth order & $1.06(0.95,1.18)$ & 0.314 & & \\
\hline
\end{tabular}

${ }^{\text {a }} H L A-D Q B 1 * 02 / D Q B 1 * 03: 02$ stratification and therefore included both high and lower risk HLA groups. The similar finding of significantly higher rates of multiple autoantibodies when the father had type 1 diabetes in our cohort of young children with a first-degree relative with type 1 diabetes and increased HLA-conferred genetic risk suggests that HLA-conferred genetic risk also is not the explanation of the observed differences.

Although different rates of progression to type 1 diabetes have been reported depending on which autoantibody appears first [27], we observed no specific difference in the first-
Table 3 HR (95\% CI) from univariate and multivariate Cox time to the development type 1 diabetes model

\begin{tabular}{|c|c|c|c|c|}
\hline \multirow[t]{2}{*}{ Variable } & \multicolumn{2}{|l|}{ Univariate models } & \multicolumn{2}{|c|}{ Multivariate model } \\
\hline & $\operatorname{HR}(95 \% \mathrm{CI})$ & $\begin{array}{l}p \\
\text { value }\end{array}$ & $\operatorname{HR}(95 \% \mathrm{CI})$ & $\begin{array}{l}p \\
\text { value }\end{array}$ \\
\hline Family history from mother & $0.43(0.29,0.65)$ & $<0.001$ & $0.58(0.36,0.93)$ & 0.023 \\
\hline Family history from father & $0.62(0.41,0.93)$ & 0.021 & $0.67(0.41,1.09)$ & 0.104 \\
\hline HLA risk $1^{\mathrm{a}}$ & $2.35(1.71,3.24)$ & $<0.001$ & $2.21(1.54,3.16)$ & $<0.001$ \\
\hline Height velocity ( $z$ score) & $1.38(1.07,1.78)$ & 0.012 & $1.41(1.10,1.82)$ & 0.007 \\
\hline Birth length & $1.01(0.95,1.07)$ & 0.732 & & \\
\hline Weight velocity, $z$ score & $1.40(1.06,1.84)$ & 0.017 & & \\
\hline Male sex & $1.07(0.78,1.46)$ & 0.682 & & \\
\hline Birthweight & $0.99(0.74,1.31)$ & 0.930 & & \\
\hline Gestational age, weeks & $1.03(0.94,1.14)$ & 0.539 & & \\
\hline \multicolumn{5}{|l|}{ Region of origin } \\
\hline Northern Europe & $1.09(0.77,1.56)$ & 0.625 & & \\
\hline Central Europe & $1.02(0.62,1.67)$ & 0.936 & & \\
\hline Vaginal delivery & $1.28(0.93,1.77)$ & 0.132 & & \\
\hline $\begin{array}{l}\text { Treatment group assignment, casein hydrosylate } \\
\text { formula }\end{array}$ & $1.06(0.78,1.45)$ & 0.717 & & \\
\hline Exclusive breastfeeding duration & $1.05(0.98,1.13)$ & 0.183 & & \\
\hline Birth order & $1.08(0.93,1.25)$ & 0.307 & & \\
\hline
\end{tabular}

${ }^{\text {a }} H L A-D Q B 1 * 02 / D Q B 1 * 03: 02$ 
appearing antibody according to type for a first-degree relative with diabetes in this large cohort. The German BABY-DIAB study [26] also reported no specific order in which autoantibody developed first according to which relative had diabetes. Hence this lower risk associated with being born to a mother with type 1 diabetes is not explained by a differing pattern in which antibody appears first.

Multiple studies, including a recent meta-analysis, have reported a link between greater birthweight and the risk of type 1 diabetes [8] or islet cell autoimmunity [10]. In contrast, Bonifacio et al [25] reported that in infants of a mother with type 1 diabetes, those in the lower and upper tertiles for birthweight as well as the presence of a moderately elevated third-trimester maternal $\mathrm{HbA}_{1 \mathrm{c}}$, had a lower risk of autoimmunity to beta cells. In our cohort, children of mothers with type 1 diabetes had greater birthweight and an earlier gestational age but no relationship was shown between their birthweight and risk of multiple autoantibodies. Hence, in our study, the relative protective effect provided by having a mother with type 1 diabetes, compared with a father or sibling with type 1 diabetes, seems not to be mediated through birthweight.

The accelerator hypothesis [28] suggests that the increasing incidence rate of type 1 diabetes is related to increasing rates of childhood obesity and insulin resistance. In the Australian BABY-DIAB study [11], weight and BMI $z$ score were found to be associated with higher rates of islet autoimmunity. Rapid linear growth during infancy has been identified as a risk factor for the development of autoimmunity and type 1 diabetes [11-13, 29]. A recent report from the TEDDY cohort found an increased risk of progression from autoimmunity to type 1 diabetes with lower height growth in infancy but increased weight gain and height gain in early childhood [30]. Similarly, we found that increased birth length and linear height velocity $z$ scores in the first 2 years of life were independently associated with the development of multiple autoantibodies. Although the TRIGR Study Group [31] had previously reported higher BMI at 5 years in Canadian girls born to a mother with type 1 diabetes, we did not find any significant differences in BMI at 24 months between the three groups based on the family history in the current analysis.

In this analysis, we also found a significant difference in the risk of developing type 1 diabetes in children born to mothers with type 1 diabetes compared with children with fathers or siblings with type 1 diabetes. Previously, the TRIGR Study Group [32] reported that there was no significant effect of type of family history on the development of type 1 diabetes in the TRIGR cohort. However, that analysis compared children with a parent only (mother and fathers were combined) to those with sibling only or those with an affected sibling and parent.

This study has some strengths. Although the TEDDY study group [33] recruited more than 8600 children, only about $10 \%$ were from a first-degree-relative family, which remains fewer than TRIGR $(n=2074)$. Our cohort, including 277 children who developed multiple beta cell autoantibodies, is one of the largest to date to explore the influence of type of family history on the development of islet cell autoimmunity. The inclusion criteria were based on presence of a firstdegree relative with type 1 diabetes and genetic (HLA) risk stratification at birth in an international multicentre, rather than country-specific, study. Data on five different autoantibodies serially measured through a follow-up period allowed for new comparisons of both type and timing of autoantibodies between the three family history groupings.

Some limitations should be considered when interpreting our results. The study presented here is based on a posteriori analysis, which cannot allow us to fully differentiate HLA effect from family history effect. The intentional wide geographical and ethnic background of the study population may have introduced significant environmental variables that could have influenced the results. We only analysed growth data from the first 24 months of life. It is possible that later growth velocity has more impact on progression to type 1 diabetes. However, this analysis was beyond the scope of this paper. Another point to consider when comparing our results with those of other studies is the definition used for multiple antibodies: defined as two or more autoantibodies present in each sample of IAA, GADA, IA-2A, ICA and ZnT8A. Some studies define this as at least two different time points with positive results and others have not included ICA in this definition due to concerns of overlapping measurements with GADA and IA-2A. The definition used in this paper was set at the start of the TRIGR study and has been used consistently in all its publications. Hence, the results presented may not be comparable with those of studies wherein a different definition of multiple antibodies had been used.

In the TRIGR cohort, children born to a mother with type 1 diabetes had a slightly lower but statistically significant risk of developing beta cell autoimmunity than those born with a father or a sibling with type 1 diabetes. However, difference in risk of developing autoantibodies based on family history type was not linked to differences in birthweight or growth (height velocity or BMI within the first 2 years of life). Ongoing efforts should likely focus on other genetic and epigenetic risks factors, as well as immunological mechanisms, to further advance our ability to identify those at risk of developing multiple antibodies and progressing to type 1 diabetes.

Acknowledgements We thank the families who participated in TRIGR for their long-term commitment to the study. Parts of this work was presented at the 43rd Annual Meeting of the International Society for Pediatric and Adolescent Diabetes (ISPAD) meeting held in Innsbruck, Austria on 18-21 October 2017.

Data availability The dataset used for these analyses can be requested by e-mailing TRIGR@epi.usf.edu. 
Funding Open access funding provided by University of Helsinki including Helsinki University Central Hospital. This work was supported by grants HD040364, HD042444 and HD051997 from the Eunice Kennedy Shriver National Institute of Child Health and Human Development and the Special Statutory Funding Program for Type 1 diabetes Research administered by the National Institute of Diabetes and Digestive and Kidney Diseases, National Institutes of Health (the content is solely the responsibility of the authors and does not necessarily represent the official views of the National Institutes of Health), Canadian Institutes of Health Research, the JDRF International, the Finnish Academy of Sciences, the Commission of the European Communities (specific RTD programme 'Quality of Life and management of Living Resources', contract number QLK1-2002-00372 'Diabetes Prevention'; it does not reflect its views and in no way anticipates the Commission's future policy in this area), and the EFSD/JDRF/Novo Nordisk Focused Research Grant. The study formulas were provided free of charge by Mead Johnson Nutrition.

Authors' relationships and activities The authors declare that there are no relationships or activities that might bias, or be perceived to bias, their work.

Contribution statement All co-authors were an integral part of acquisition of the data and interpretation of the analysis. All co-authors have seen and approved the final version of the manuscript. More specific contributions are as follows: DP, AMN and DC wrote the manuscript, planned the analysis, researched data and contributed to discussion; DJB and SMV researched data, contributed to discussion and critically reviewed/ edited the manuscript; MK was responsible for the autoantibody analyses, contributed to discussion and critically reviewed/edited the manuscript; and JL and JI contributed to discussion and critically reviewed/edited the manuscript. MK, on behalf of the TRIGR Investigators, takes full responsibility for the work, including the study design, access to data and the decision to submit and publish the manuscript.

Open Access This article is licensed under a Creative Commons Attribution 4.0 International License, which permits use, sharing, adaptation, distribution and reproduction in any medium or format, as long as you give appropriate credit to the original author(s) and the source, provide a link to the Creative Commons licence, and indicate if changes were made. The images or other third party material in this article are included in the article's Creative Commons licence, unless indicated otherwise in a credit line to the material. If material is not included in the article's Creative Commons licence and your intended use is not permitted by statutory regulation or exceeds the permitted use, you will need to obtain permission directly from the copyright holder. To view a copy of this licence, visit http://creativecommons.org/licenses/by/4.0/.

\section{References}

1. Karvonen M, Viik-Kajander M, Moltchanova E, Libman I, LaPorte R, Tuomilehto J (2000) Incidence of childhood type 1 diabetes worldwide. Diabetes Mondiale (DiaMond) Project Group. Diabetes Care 23(10):1516-1526. https://doi.org/10.2337/diacare. 23.10.1516

2. Patterson CC, Dahlquist GG, Gyurus E, Green A, Soltesz G, EURODIAB Study Group (2009) Incidence trends for childhood type 1 diabetes in Europe during 1989-2003 and predicted new cases 2005-20: a multicentre prospective registration study. Lancet 373(9680):2027-2033. https://doi.org/10.1016/S01406736(09)60568-7

3. Gale EAM (2002) The rise of childhood type 1 diabetes in the 20th century. Diabetes 51(12):3353-3361. https://doi.org/10.2337/ diabetes.51.12.3353
4. Chen YL, Huang YC, Qiao YC et al (2017) Climates on incidence of childhood type 1 diabetes mellitus in 72 countries. Sci Rep 7(1): 12810. https://doi.org/10.1038/s41598-017-12954-8

5. Ludvigsson J (2017) Increasing Incidence but decreasing awareness of type 1 diabetes in Sweden. Diabetes Care 40(10):e143e144. https://doi.org/10.2337/dc17-1175

6. DiMeglio LA, Evans-Molina C, Oram RA (2018) Type 1 diabetes. Lancet 391(10138):2449-2462. https://doi.org/10.1016/S01406736(18)31320-5

7. Gale EA, Gillespie KM (2001) Diabetes and gender. Diabetologia 44(1):3-15. https://doi.org/10.1007/s001250051573

8. Cardwell CR, Stene LC, Joner G et al (2010) Birthweight and the risk of childhood-onset type 1 diabetes: a meta-analysis of observational studies using individual patient data. Diabetologia 53(4): 641-651. https://doi.org/10.1007/s00125-009-1648-5

9. Stene LC, Magnus P, Lie RT, Sovik O, Joner G, Norwegian childhood Diabetes Study Group (2001) Birth weight and childhood onset type 1 diabetes: population based cohort study. BMJ 322(7291):889-892. https://doi.org/10.1136/bmj.322.7291.889

10. Larsson HE, Lynch K, Lernmark B et al (2005) Diabetes-associated HLA genotypes affect birthweight in the general population. Diabetologia 48(8):1484-1491. https://doi.org/10.1007/s00125005-1813-4

11. Couper JJ, Beresford S, Hirte C et al (2009) Weight gain in early life predicts risk of islet autoimmunity in children with a first-degree relative with type 1 diabetes. Diabetes Care 32(1):94-99. https:// doi.org/10.2337/dc08-0821

12. Lamb MM, Yin X, Zerbe GO et al (2009) Height growth velocity, islet autoimmunity and type 1 diabetes development: the Diabetes Autoimmunity Study in the Young. Diabetologia 52(10):2064 2071. https://doi.org/10.1007/s00125-009-1428-2

13. Larsson HE, Hansson G, Carlsson A et al (2008) Children developing type 1 diabetes before 6 years of age have increased linear growth independent of HLA genotypes. Diabetologia 51(9):16231630. https://doi.org/10.1007/s00125-008-1074-0

14. Hypponen E, Virtanen SM, Kenward MG, Knip M, Akerblom HK, Childhood Diabetes in Finland Study Group (2000) Obesity, increased linear growth, and risk of type 1 diabetes in children. Diabetes Care 23(12):1755-1760. https://doi.org/10.2337/diacare. 23.12.1755

15. Krischer JP, Liu X, Lernmark A et al (2017) The influence of type 1 diabetes genetic susceptibility regions, age, sex, and family history on the progression from multiple autoantibodies to type 1 diabetes: a TEDDY study report. Diabetes 66(12):3122-3129. https://doi. org/10.2337/db17-0261

16. Elding Larsson H, Vehik K, Haller MJ et al (2016) Growth and risk for islet autoimmunity and progression to type 1 diabetes in early childhood: The Environmental Determinants of Diabetes in the Young Study. Diabetes 65(7):1988-1995. https://doi.org/10.2337/ db15-1180

17. Krischer JP, Lynch KF, Lernmark A et al (2017) Genetic and environmental interactions modify the risk of diabetes-related autoimmunity by 6 years of age: The TEDDY study. Diabetes Care 40(9): 1194-1202. https://doi.org/10.2337/dc17-0238

18. TRIGR Study Group (2007) Study design of the Trial to Reduce IDDM in the Genetically at Risk (TRIGR). Pediatr Diabetes 8(3): 117-137. https://doi.org/10.1111/j.1399-5448.2007.00239.x

19. Knip M, Akerblom HK, Becker D et al (2014) Hydrolyzed infant formula and early beta-cell autoimmunity: a randomized clinical trial. JAMA 311(22):2279-2287. https://doi.org/10.1001/jama. 2014.5610

20. Kuczmarski RJ, Ogden CL, Guo SS et al (2002) 2000 CDC Growth charts for the United States: methods and development. Vital Health Stat 11(246):1-190 
21. Knip M, Virtanen SM, Seppa K et al (2010) Dietary intervention in infancy and later signs of $\beta$-cell autoimmunity. N Engl J Med 363(20):1900-1908. https://doi.org/10.1056/NEJMoa1004809

22. Turtinen M, Harkonen T, Parkkola A, Ilonen J, Knip M, Finnish Pediatric Diabetes Register (2018) Sex as a determinant of type 1 diabetes at diagnosis. Pediatr Diabetes 19(7):1221-1228. https:// doi.org/10.1111/pedi.12697

23. Yu L, Chase HP, Falorni A, Rewers M, Lernmark A, Eisenbarth GS (1995) Sexual dimorphism in transmission of expression of islet autoantibodies to offspring. Diabetologia 38(11):1353-1357. https://doi.org/10.1007/bf00401769

24. Hippich M, Beyerlein A, Hagopian WA et al (2019) Genetic contribution to the divergence in type 1 diabetes risk between children from the general population and children from affected families. Diabetes 68(4):847-857. https://doi.org/10.2337/db18-0882

25. Bonifacio E, Pfluger M, Marienfeld S, Winkler C, Hummel M, Ziegler AG (2008) Maternal type 1 diabetes reduces the risk of islet autoantibodies: relationships with birthweight and maternal $\mathrm{HbA}(1 \mathrm{c})$. Diabetologia 51(7):1245-1252. https://doi.org/10.1007/ s00125-008-1022-z

26. Roll U, Christie MR, Fuchtenbusch M, Payton MA, Hawkes CJ, Ziegler AG (1996) Perinatal autoimmunity in offspring of diabetic parents. The German Multicenter BABY-DIAB study: detection of humoral immune responses to islet antigens in early childhood. Diabetes 45(7):967-973. https://doi.org/10.2337/diab.45.7.967

27. Ilonen J, Hammais A, Laine AP et al (2013) Patterns of beta-cell autoantibody appearance and genetic associations during the first years of life. Diabetes 62(10):3636-3640. https://doi.org/10.2337/ db13-0300
28. Wilkin TJ (2001) The accelerator hypothesis: weight gain as the missing link between type 1 and type 11 diabetes. Diabetologia 44: 914-922

29. Johansson C, Samuelsson U, Ludvigsson J (1994) A high weight gain early in life is associated with an increased risk of type 1 (insulin-dependent) diabetes mellitus. Diabetologia 37(1):91-94. https://doi.org/10.1007/BF00428783

30. Liu X, Vehik K, Huang Y et al (2020) Distinct growth phases in early life associated with the risk of type 1 diabetes: the TEDDY study. Diabetes Care 43(3):556-562. https://doi.org/10.2337/dc191670

31. Nucci AM, Becker DJ, Virtanen SM et al (2012) Growth differences between North American and European children at risk for type 1 diabetes. Pediatr Diabetes 13(5):425-431. https://doi.org/10. 1111/j.1399-5448.2011.00840.x

32. Writing Group for the TRIGR Study Group, Knip M, Akerblom HK et al (2018) Effect of hydrolyzed infant formula vs conventional formula on risk of type 1 diabetes: the TRIGR randomized clinical trial. JAMA 319(1):38-48. https://doi.org/10.1001/jama.2017. 19826

33. Krischer JP, Liu X, Vehik K et al (2019) Predicting islet cell autoimmunity and type 1 diabetes: an 8-year TEDDY study progress report. Diabetes Care 42(6):1051-1060. https://doi.org/10.2337/ dc18-2282

Publisher's note Springer Nature remains neutral with regard to jurisdictional claims in published maps and institutional affiliations.

\section{Affiliations}

\section{Danièle Pacaud ${ }^{1}$ (1) - Anita M. Nucci ${ }^{2}$ (D) $\cdot$ David Cuthbertson $^{3}$ (D) Dorothy J. Becker ${ }^{4}$ (D) $\cdot$ Suvi M. Virtanen ${ }^{5,6,7,8}$ (D) Johnny Ludvigsson ${ }^{9}$ (D) . Jorma llonen ${ }^{10,11}$ (D) $\cdot$ Mikael Knip ${ }^{12,13}$ (D) . the TRIGR investigators}

1 Department of Pediatrics, Alberta Children's Hospital, University of Calgary, Calgary, AB, Canada

2 Department of Nutrition, Georgia State University, Atlanta, GA, USA

3 Pediatrics Epidemiology Center, University of South Florida, Tampa, FL, USA

4 Division of Endocrinology, University of Pittsburgh and UPMC Children's Hospital of Pittsburgh, Pittsburgh, PA, USA

5 Public Health Promotion Unit, National Institute for Health and Welfare, Helsinki, Finland

6 Faculty of Social Sciences/Health, Tampere University, Tampere, Finland

7 Center for Child Health Research, Tampere University, Tampere, Finland
8 Research, Development and Innovation Centre, Tampere University Hospital, Tampere, Finland

9 Crown Princess Victoria Children's Hospital, Region Östergötland and Division of Pediatrics, Department of Clinical Experimental Medicine, Linkoping University, Linkoping, Sweden

10 Immunogenetics Laboratory, Institute of Biomedicine, University of Turku, Turku, Finland

11 Clinical Microbiology, Turku University Hospital, Turku, Finland

12 Pediatric Research Center, Children's Hospital, University of Helsinki and Helsinki University Hospital, Helsinki, Finland

13 Research Program for Clinical and Molecular Metabolism, Faculty of Medicine, University of Helsinki, Helsinki, Finland 